\title{
APPROXIMATE INVERSE SYSTEMS OF COMPACTA AND COVERING DIMENSION
}

\author{
Sibe MARdešíć ANd LeONARd R. Rubin
}

\begin{abstract}
Approximate inverse systems of metric compacta are introduced and studied. The bonding maps in these systems commute only up to certain controlled values. With every such system $\mathbf{X}=\left(X_{a}, \varepsilon_{a}, p_{a a^{\prime}}, A\right)$ are associated a limit space $X$ and projections $p_{a}: X \rightarrow X_{a}$. A compact Hausdorff space $X$ has covering dimension $\operatorname{dim} X \leq n$ if and only if it can be obtained as the limit of an approximate inverse system of compact polyhedra of dimension $\leq n$. The analogous statement for usual inverse systems is known to be false.
\end{abstract}

1. Introduction. An inverse system of spaces $\mathbf{X}=\left(X_{a}, p_{a a^{\prime}}, A\right)$, in the usual sense, consists of a directed set $A$, spaces $X_{a}, a \in A$, and maps $p_{a a^{\prime}}: X_{a^{\prime}} \rightarrow X_{a}, a \leq a^{\prime}$, such that $p_{a a}=\mathrm{id}, p_{a a^{\prime}} p_{a^{\prime} a^{\prime \prime}}=p_{a a^{\prime \prime}}$, $a \leq a^{\prime} \leq a^{\prime \prime}$. The (usual) inverse limit of $X$ is the subspace $X \subseteq \prod X_{a}$ which consists of all points $x=\left(x_{a}\right) \in \prod X_{a}$ such that $p_{a a^{\prime}}\left(x_{a^{\prime}}\right)=x_{a}$ whenever $a \leq a^{\prime}$. Projections $p_{a}: X \rightarrow X_{a}$ are just the restrictions $p_{a}=\pi_{a} \mid X$ of the projections $\pi_{a}: \prod X_{a} \rightarrow X_{a}$.

It is well known that the inverse limit of an inverse system of nonempty compact spaces $X_{a}$ is a non-emepty compact space $X$. If the covering dimension $\operatorname{dim} X_{a} \leq n, a \in A$, then also $\operatorname{dim} X \leq n$. In particular, a limit of compact polyhedra $P_{a}$ with $\operatorname{dim} P_{a} \leq n$ is a compact Hausdorff space with $\operatorname{dim} X \leq n$.

On the other hand, every compact Hausdorff space $X$ is the limit of an inverse system of compact polyhedra $P_{a}$ [1]. If $X$ is a compact metric space and $\operatorname{dim} X \leq n$, one can obtain $X$ as the limit of an inverse sequence of compact polyhedra $P_{a}$ with $\operatorname{dim} P_{a} \leq n$ ([2], also see [4]). However, the analogous statement for compact Hausdorff spaces is false as shown in 1958 independently by $S$. Mardešic [4] and B. A. Pasynkov [6]. These authors produced examples of compact Hausdorff spaces $X$ with $\operatorname{dim} X=1$ which cannot be represented as inverse limits of inverse systems of compact polyhedra of dimension $\leq 1$. Further examples of this type were given by Mardešić in [3] and Pasynkov in [7]. Recently, Mardešić and T. Watanabe [5] have shown that a 1-dimensional compact Hausdorff space considered by 
Pasynkov in [7] is not even a limit of a system of compact ANR's of dimension $\leq 1$.

In the present paper we define approximate inverse systems of metric compacta and their limits, and we prove that compact Hausdorff spaces $X$ of $\operatorname{dim} X \leq n$ coincide with limits of approximate inverse systems of compact polyhedra of dimension $\leq n$ (see $\S 6$, Theorem 5). Our approximate inverse systems differ from the usual inverse systems in that we do not insist on the commutativity requirement $p_{a a^{\prime}} p_{a^{\prime} a^{\prime \prime}}=p_{a a^{\prime \prime}}, a \leq a^{\prime} \leq a^{\prime \prime}$; rather we allow the two maps $p_{a a^{\prime}} p_{a^{\prime} a^{\prime \prime}}$ and $p_{a a^{\prime \prime}}$ to differ, but in a controlled way. In particular, for sufficiently large $a^{\prime}, a^{\prime \prime}$ the difference becomes arbitrarily small (see $\S 2$, Definition 1).

\section{Approximate inverse systems, basic definitions.}

Definition 1. An approximate inverse system $\mathbf{X}=\left(X_{a}, \varepsilon_{a}, p_{a a^{\prime}}, A\right)$ of metric compacta consists of the following: an ordered set $(A, \leq)$ which is directed and has no maximal element; for each $a \in A$, a compact metric space $X_{a}$ with metric $d$ and a real number $\varepsilon_{a}>0$; for each pair $a \leq a^{\prime}$ from $A$, a mapping $p_{a a^{\prime}}: X_{a^{\prime}} \rightarrow X_{a}$. Moreover the following three conditions must be satisfied:

(A1) $d\left(p_{a_{1} a_{2}} p_{a_{2} a_{3}}, p_{a_{1} a_{3}}\right) \leq \varepsilon_{a_{1}}, a_{1} \leq a_{2} \leq a_{3}, p_{a a}=$ id.

(A2) $(\forall a \in A)(\forall \eta>0)\left(\exists a^{\prime} \geq a\right)\left(\forall a_{2} \geq a_{1} \geq a^{\prime}\right) d\left(p_{a a_{1}} p_{a_{1} a_{2}}, p_{a a_{2}}\right)$ $\leq \eta$.

(A3) $(\forall a \in A)(\forall \eta>0)\left(\exists a^{\prime} \geq a\right)\left(\forall a^{\prime \prime} \geq a^{\prime}\right)\left(\forall x, x^{\prime} \in X_{a^{\prime \prime}}\right) d\left(x, x^{\prime}\right)$ $\leq \varepsilon_{a^{\prime \prime}} \Rightarrow d\left(p_{a a^{\prime \prime}}(x), p_{a a^{\prime \prime}}\left(x^{\prime}\right)\right) \leq \eta$.

REMARK 1. If $a^{\prime}$ satisfies (A2), then any $a_{1}^{\prime} \geq a^{\prime}$ also does. The same applies to (A3). Therefore, one can find $a^{\prime}$ which simultaneously satisfies (A2) and (A3) (even for a finite collection of $a$ 's and $\eta$ 's).

Remark 2. If $\mathbf{X}=\left(X_{a}, p_{a a^{\prime}}, A\right)$ is a usual inverse system of metric compacta, $A$ has no maximal element and is cofinite (every element has only finitely many predecessors), one can define numbers $\varepsilon_{a}>0$ so that $\left(X_{a}, \varepsilon_{a}, p_{a a^{\prime}}, A\right)$ is an approximate inverse system.

Indeed, let $\left|a^{\prime}\right|$ denote the number of elements $a$ of $A$ such that $a<a^{\prime}$ (i.e., $a \leq a^{\prime}$ and $a \neq a^{\prime}$ ). Since $A$ is infinite, it suffices to define numbers $\varepsilon_{a}$ such that for each $x, x^{\prime} \in X_{a^{\prime}}$

$$
d\left(x, x^{\prime}\right) \leq \varepsilon_{a^{\prime}}
$$

implies

$$
d\left(p_{a a^{\prime}}(x), p_{a a^{\prime}}\left(x^{\prime}\right)\right) \leq 1 / 2^{\left|a^{\prime}\right|}, \quad a<a^{\prime} .
$$


This can be done by induction on $|a|$, using uniform continuity of the maps $p_{a a^{\prime}}$ and the assumption that $A$ is cofinite.

With every approximate system $\mathbf{X}=\left(X_{a}, \varepsilon_{a}, p_{a a^{\prime}}, A\right)$ we associate its limit $X=\lim \mathbf{X}$, which is a subspace of $\prod X_{a}$.

Definition 2. A point $x=\left(x_{a}\right) \in \prod_{a \in A} X_{a}$ belongs to $X=\lim \mathbf{X}$ provided the following condition is satisfied.

$$
(\forall a \in A)(\forall n>0)\left(\exists a^{\prime} \geq a\right)\left(\forall a^{\prime \prime} \geq a^{\prime}\right) \quad d\left(x_{a}, p_{a a^{\prime \prime}}\left(x_{a^{\prime \prime}}\right)\right) \leq \eta .
$$

Condition (L) can also be stated as

$$
(\forall a \in A) \quad x_{a}=\lim _{a^{\prime \prime}} p_{a a^{\prime \prime}}\left(x_{a^{\prime \prime}}\right) .
$$

We refer to points of $X$ as threads. We also consider maps $p_{a}: X \rightarrow$ $X_{a}, a \in A$, which are defined as restrictions

$$
p_{a}=\pi_{a} \mid X
$$

of the projections $\pi_{a}: \prod X_{a} \rightarrow X_{a}$.

Proposition 1. Let $\mathbf{X}=\left(X_{a}, \varepsilon_{a}, p_{a a^{\prime}}, A\right)$ be an approximate inverse system. If $\mathbf{X}$ is commutative, i.e., $p_{a a^{\prime}} p_{a^{\prime} a^{\prime \prime}}=p_{a a^{\prime \prime}}, a \leq a^{\prime} \leq a^{\prime \prime}$, then the limit $X=\lim \mathbf{X}$ as defined in Definition 2, coincides with the usual limit of $\mathbf{X}=\left(X_{a}, p_{a a^{\prime}}, A\right)$.

Proof. Let $x=\left(x_{a}\right) \in \lim \mathbf{X}$, let $a_{1} \leq a_{2}$ and $\eta>0$. Then by (L), for all sufficiently large $a^{\prime \prime} \geq a_{1}$ one has

$$
d\left(x_{a_{1}}, p_{a_{1} a^{\prime \prime}}\left(x_{a^{\prime \prime}}\right)\right) \leq n / 2 \text {. }
$$

Let $\delta>0$ be chosen so that $d\left(x, x^{\prime}\right) \leq \delta$ implies $d\left(p_{a_{1} a_{2}}(x), p_{a_{1} a_{2}}\left(x^{\prime}\right)\right) \leq$ $\eta / 2$. Then by (L), for all sufficiently large $a^{\prime \prime} \geq a_{2}$,

$$
d\left(x_{a_{2}}, p_{a_{2} a^{\prime \prime}}\left(x_{a^{\prime \prime}}\right)\right) \leq \delta
$$

and therefore

$$
d\left(p_{a_{1} a_{2}}\left(x_{a_{2}}\right), p_{a_{1} a_{2}} p_{a_{2} a^{\prime \prime}}\left(x_{a^{\prime \prime}}\right)\right) \leq \eta / 2 .
$$

Since $p_{a_{1} a_{2}} p_{a_{2} a^{\prime \prime}}=p_{a_{1} a^{\prime \prime}},(4)$ and (6) imply

$$
d\left(p_{a_{1} a_{2}}\left(x_{a_{2}}\right), x_{a_{1}}\right) \leq \eta \text {. }
$$

Since $\eta$ was arbitrary, (7) shows that $p_{a_{1} a_{2}}\left(x_{a_{2}}\right)=x_{a_{1}}$, so that $x=\left(x_{a}\right)$ is a point of the usual limit. The reverse inclusion is obvious.

Remark 2 and Proposition 1 show that our "ap sroximate" notions extend the usual ones. 
Proposition 2. Let $\mathbf{X}=\left(X_{a}, \varepsilon_{a}, p_{a a^{\prime}}, A\right)$ be an approximate system and let $\varepsilon_{a}^{\prime}>0$ be numbers such that $\varepsilon_{a}^{\prime} \leq \varepsilon_{a}$. Then there is an ordering $\leq^{\prime}$ of $A$ such that $A^{\prime}=\left(A, \leq^{\prime}\right)$ is directed and $a_{1} \leq^{\prime} a_{2}$ implies $a_{1} \leq a_{2}$. Moreover, $\mathbf{X}^{\prime}=\left(X_{a}, \varepsilon_{a}^{\prime}, p_{a a^{\prime}}, A^{\prime}\right)$ is also an approximate system and the limits $X=\lim \mathbf{X}$ and $X^{\prime}=\lim \mathbf{X}^{\prime}$ coincide.

Proposition 2 shows that one can diminish the numbers $\varepsilon_{a}$ below preassigned levels and still preserve the limit space.

Proof. We put $a<^{\prime} a^{\prime}$ if $a<a^{\prime}$ and $a^{\prime}$ satisfies (A2) and (A3) for $\eta=\varepsilon_{a}^{\prime}$. We put $a \leq^{\prime} a^{\prime}$ if $a<^{\prime} a^{\prime}$ or $a=a^{\prime}$. It is readily seen that $\leq^{\prime}$ is a new ordering of $A$ and that $A^{\prime}=\left(A, \leq^{\prime}\right)$ is directed. Moreover, $a_{1} \leq a_{2}$ implies $a_{1} \leq a_{2}$.

It is also easy to see that $\mathbf{X}^{\prime}$ is an approximate system and $X \subseteq X^{\prime}$. To show this we repeatedly use the following facts. Every $a \in A$ admits an $a^{\prime} \in A$ such that $a<^{\prime} a^{\prime}$. If $a_{1} \leq a_{2}$, then there is an $a_{2}^{\prime} \geq a_{2}$ such that $a_{1} \leq a_{2}^{\prime}$. If $a_{1} \leq a_{2}$ and $a_{2} \leq a_{3}$, then $a_{1} \leq a_{3}$.

Now we will prove the reverse inclusion $X^{\prime} \subseteq X$. Let $x=\left(x_{a}\right) \in X^{\prime}$. Consider $a \in A$ and $\eta>0$ and choose $a^{\prime}$ in accordance with (L) for $X^{\prime}, a$ and $\eta / 5$. Note that $a \leq^{\prime} a^{\prime}$. We will also assume that $a^{\prime}$ is so large that (A2) and (A3) hold for $\mathbf{X}, a$ and $\eta / 5$.

We claim that $a^{\prime}$ also satisfies (L) for $\mathbf{X}, a$ and $\eta$, so that $x \in X$. Indeed, let $a_{1} \geq a^{\prime}$. Since $x \in X^{\prime}$, one can choose $a^{\prime \prime}$ so large that $a^{\prime} \leq^{\prime} a^{\prime \prime}, a_{1} \leq^{\prime} a^{\prime \prime}$, and

$$
\begin{gathered}
d\left(x_{a}, p_{a a^{\prime \prime}}\left(x_{a^{\prime \prime}}\right)\right) \leq \eta / 5, \\
d\left(p_{a a^{\prime}} p_{a^{\prime} a_{1}} p_{a_{1} a^{\prime \prime}}\left(x_{a^{\prime \prime}}\right), p_{a a^{\prime}} p_{a^{\prime} a_{1}}\left(x_{a_{1}}\right)\right) \leq \eta / 5 .
\end{gathered}
$$

Formula (9) is obtained by first choosing a $\delta>0$ so small that $p_{a a^{\prime}} p_{a^{\prime} a_{1}}$ maps $\delta$-near points to $\eta / 5$ near points, and then applying $(\mathrm{L})$ to $\mathbf{X}^{\prime}$, $a_{1}$ and $\delta$.

Note that

$$
d\left(p_{a^{\prime} a^{\prime \prime}}\left(x_{a^{\prime \prime}}\right), p_{a^{\prime} a_{1}} p_{a_{1} a^{\prime \prime}}\left(x_{a^{\prime \prime}}\right)\right) \leq \varepsilon_{a^{\prime}} .
$$

Therefore by the choice of $a^{\prime}$, we obtain respectively from (A3), (A2), (A2),

$$
\begin{gathered}
d\left(p_{a a^{\prime}} p_{a^{\prime} a^{\prime \prime}}\left(x_{a^{\prime \prime}}\right), p_{a a^{\prime}} p_{a^{\prime} a_{1}} p_{a_{1} a^{\prime \prime}}\left(x_{a^{\prime \prime}}\right)\right) \leq \eta / 5, \\
d\left(p_{a a^{\prime}} p_{a^{\prime} a^{\prime \prime}}\left(x_{a^{\prime \prime}}\right), p_{a a^{\prime \prime}}\left(x_{a^{\prime \prime}}\right)\right) \leq \eta / 5, \\
d\left(p_{a a_{1}}\left(x_{a_{1}}\right), p_{a a^{\prime}} p_{a^{\prime} a_{1}}\left(x_{a_{1}}\right)\right) \leq \eta / 5 .
\end{gathered}
$$


Clearly, (8), (12), (11), (9) and (13) imply

$$
d\left(x_{a}, p_{a a_{1}}\left(x_{a_{1}}\right)\right) \leq \eta,
$$

which is the desired inequality.

\section{The limit space is non-empty.}

THEOREM 1. If in an approximate system $\mathbf{X}=\left(X_{a}, \varepsilon_{a}, p_{a a^{\prime}}, A\right)$, all $X_{a} \neq \varnothing$, then also $X=\lim \mathbf{X} \neq \varnothing$.

In order to prove this theorem we first define prethreads of an approximate inverse system.

Definition 3. Let $\mathbf{X}$ be an approximate system. A point $x=\left(x_{a}\right) \in$ $\prod_{a \in A} X_{a}$ is called a prethread of $\mathbf{X}$ provided for every pair $a \leq a^{\prime}$ one has

$$
d\left(x_{a}, p_{a a^{\prime}}\left(x_{a^{\prime}}\right)\right) \leq \varepsilon_{a} .
$$

Lemma 1. If each $X_{a} \neq \varnothing$, then the set $X^{p}$ of all prethreads is nonempty.

Proof. For each pair $a \leq a^{\prime}$ we define a set $X_{a a^{\prime}} \subseteq \prod_{a \in A} X_{a}$ by putting $x=\left(x_{a}\right) \in X_{a a^{\prime}}$ whenever (1) holds for the pair $\left(a, a^{\prime}\right)$. Clearly each $X_{a a^{\prime}}$ is a closed subset of $\prod X_{a}$. Moreover, the set of all prethreads $X^{p}$ satisfies

$$
X^{p}=\bigcap_{a \leq a^{\prime}} X_{a a^{\prime}}
$$

It therefore suffices to prove that the collection $\left\{X_{a a^{\prime}}: a \leq a^{\prime}\right\}$ has the finite intersection property.

If $a_{1} \leq a_{1}^{\prime}, \ldots, a_{n} \leq a_{n}^{\prime}$, choose $a^{\prime} \geq a_{1}^{\prime}, \ldots, a_{n}^{\prime}$. Take any $x_{a^{\prime}} \in X_{a^{\prime}}$ and define $x_{a_{t}} \in X_{a_{l}}$ and $x_{a_{l}^{\prime}} \in X_{a_{t}^{\prime}}$ by

$$
\begin{array}{ll}
x_{a_{t}}=p_{a_{t} a^{\prime}}\left(x_{a^{\prime}}\right), & i=1, \ldots, n, \\
x_{a_{t}^{\prime}}=p_{a_{i}^{\prime} a^{\prime}}\left(x_{a^{\prime}}\right), & i=1, \ldots, n .
\end{array}
$$

For $a \in A \backslash\left\{a_{1}, \ldots, a_{n}, a_{1}^{\prime}, \ldots, a_{n}^{\prime}, a^{\prime}\right\}$ choose for $x_{a}$ any point in $X_{a}$.

By (3), (4) and (A1) we have

$$
d\left(p_{a_{l} a_{l}^{\prime}}\left(x_{a_{\imath}^{\prime}}\right), x_{a_{l}}\right) \leq \varepsilon_{a_{l}}, \quad i=1, \ldots, n .
$$


This shows that

$$
x=\left(x_{a}\right) \in X_{a_{1} a_{t}^{\prime}}, \quad i=1, \ldots, n,
$$

so that $x \in X_{a_{1} a_{1}^{\prime}} \cap \cdots \cap X_{a_{n} a_{n}^{\prime}} \neq \varnothing$.

Lemma 2. Let $x=\left(x_{a}\right)$ be a prethread. Then

$$
y_{a}=\lim _{a^{\prime}} p_{a a^{\prime}}\left(x_{a^{\prime}}\right)
$$

exists for each $a \in A$. Moreover, $y=\left(y_{a}\right)$ is a thread, that is, $y \in X=$ $\lim \mathbf{X}$.

Proof. Fix $a \in A$ and consider all $a^{\prime} \geq a$. Then $\left(p_{a a^{\prime}}\left(x_{a^{\prime}}\right): a^{\prime} \geq a\right)$ is a net in $X_{a}$. We will prove that this is a Cauchy net and therefore the limit in (7) exists.

For a given $\eta>0$ choose $a^{\prime} \geq a$ so that (A2) and (A3) hold. If $a^{\prime} \leq a_{1} \leq a_{3}$, then

$$
d\left(p_{a a_{1}} p_{a_{1} a_{3}}\left(x_{a_{3}}\right), p_{a a_{3}}\left(x_{a_{3}}\right)\right) \leq \eta .
$$

Since $x$ is a prethread, we also have $d\left(x_{a_{1}}, p_{a_{1} a_{3}}\left(x_{a_{3}}\right)\right) \leq \varepsilon_{a_{1}}$, and thus

$$
d\left(p_{a a_{1}}\left(x_{a_{1}}\right), p_{a a_{1}} p_{a_{1} a_{3}}\left(x_{a_{3}}\right)\right) \leq \eta \text {. }
$$

Statements (8) and (9) yield

$$
d\left(p_{a a_{1}}\left(x_{a_{1}}\right), p_{a a_{3}}\left(x_{a_{3}}\right)\right) \leq 2 \eta .
$$

Analogously, for $a^{\prime} \leq a_{2} \leq a_{3}$ we have

$$
d\left(p_{a a_{2}}\left(x_{a_{2}}\right), p_{a a_{3}}\left(x_{a_{3}}\right)\right) \leq 2 \eta .
$$

Using directedness of A, (10) and (11), we conclude that

$$
d\left(p_{a a_{1}}\left(x_{a_{1}}\right), p_{a a_{2}}\left(x_{a_{2}}\right)\right) \leq 4 \eta, \quad \text { whenever } a^{\prime} \leq a_{1} \text { and } a^{\prime} \leq a_{2} \text {. }
$$

Consequently, $\left(p_{a a^{\prime}}\left(x_{a^{\prime}}\right): a^{\prime} \geq a\right)$ is a Cauchy net.

In order to see that (7) defines a thread $y=\left(y_{a}\right) \in X$, it suffices to notice that the application of $\lim _{a_{3}}$ to (8) yields

$$
d\left(p_{a a_{1}}\left(y_{a_{1}}\right), y_{a}\right) \leq \eta, \quad a^{\prime} \leq a_{1},
$$

which establishes property $(\mathrm{L})$ for $y$.

Theorem 1 is an immediate consequence of Lemmas 1 and 2. 


\section{The limit space is compact.}

THEOREM 2. The limit $X$ of an approximate system of compacta is a compact Hausdorff space.

Proof. It suffices to show that $X$ is a closed subset of $\prod X_{a}$. Let $y=\left(y_{a}\right) \in\left(\Pi X_{a}\right) \backslash X$. We will exhibit a neighborhood $U$ of $y$ such that $U \cap X=\varnothing$. This will prove that $\left(\prod X_{a}\right) \backslash X$ is open.

Since $y$ is not a thread of $\mathbf{X}$, there is an $a_{0} \in A$ and there is an $\eta>0$ such that for every $a^{\prime} \geq a_{0}$ there exists an $a^{\prime \prime} \geq a^{\prime}$ satisfying

$$
d\left(y_{a_{0}}, p_{a_{0} a^{\prime \prime}}\left(y_{a^{\prime \prime}}\right)\right)>\eta \text {. }
$$

Choose $a^{\prime} \geq a_{0}$ so that (A2) and (A3) hold for $a_{0}$ and $\eta / 6$. Then choose $a^{\prime \prime} \geq a^{\prime}$ so that (1) holds. Finally, define $U$ as the set of all points $x=\left(x_{a}\right) \in \prod X_{a}$ which satisfy

$$
\begin{aligned}
& d\left(x_{a_{0}}, y_{a_{0}}\right)<\eta / 3, \\
& d\left(x_{a^{\prime \prime}}, y_{a^{\prime \prime}}\right)<\varepsilon_{a^{\prime \prime}} .
\end{aligned}
$$

Clearly $U$ is an open set in $\prod X_{a}$ and $y \in U$.

We claim that $U \cap X=\varnothing$. Assume to the contrary that $x=\left(x_{a}\right) \in$ $U \cap X$. By (L) applied to $x, a_{0}$ and $\eta / 3$, for sufficiently large indexes $a^{*} \geq a^{\prime \prime}$ one has

$$
d\left(x_{a_{0}}, p_{a_{0} a^{*}}\left(x_{a^{*}}\right)\right) \leq \eta / 3 .
$$

By choosing $a^{*}$ large enough we can also obtain

$$
d\left(y_{a^{\prime \prime}}, p_{a^{\prime \prime} a^{*}}\left(x_{a^{*}}\right)\right) \leq \varepsilon_{a^{\prime \prime}}
$$

Indeed, by (3), there is a $\delta>0$ such that the $\delta$-neighborhood $N\left(x_{a^{\prime \prime}}, \delta\right) \subseteq N\left(y_{a^{\prime \prime}}, \varepsilon_{a^{\prime \prime}}\right)$. So (L) applied to $x, a^{\prime \prime}$ and $\delta / 2$ shows that $d\left(x_{a^{\prime \prime}}, p_{a^{\prime \prime} a^{*}}\left(x_{a^{*}}\right)\right)<\delta$ for $a^{*} \geq a^{\prime \prime}$ sufficiently large. However, this implies (5).

By the choice of $a^{\prime}$ (property (A3)) and (5), we see that

$$
d\left(p_{a_{0} a^{\prime \prime}} p_{a^{\prime \prime} a^{*}}\left(x_{a^{*}}\right), p_{a_{0} a^{\prime \prime}}\left(y_{a^{\prime \prime}}\right)\right) \leq \eta / 6
$$

Furthermore, by (A2),

$$
d\left(p_{a_{0} a^{\prime \prime}} p_{a^{\prime \prime} a^{*}}\left(x_{a^{*}}\right), p_{a_{0} a^{*}}\left(x_{a^{*}}\right)\right) \leq \eta / 6
$$

Now (2), (4), (7) and (6) yield

$$
d\left(y_{a_{0}}, p_{a_{0} a^{\prime \prime}}\left(y_{a^{\prime \prime}}\right)\right) \leq \eta
$$

which contradicts (1). 


\section{Covering dimension of the limit space.}

Theorem 3. Let $\mathbf{X}=\left(X_{a}, \varepsilon_{a}, p_{a a^{\prime}}, A\right)$ be an approximate system of metric compacta with limit $X$ and let $\mathscr{U}$ be an open covering of $X$. Then there exist an index $a \in A$ and an open covering $\mathscr{V}$ of $X_{a}$ such that $p_{a}^{-1}(\mathscr{V})$ refines $\mathscr{U}$.

We will first prove a lemma describing a basis for the topology of $X$.

Lemma 3. The collection of all sets of the form $p_{a}^{-1}\left(V_{a}\right)$, where $a \in A$ and $V_{a} \subseteq X_{a}$ is open, is a basis for the topology of $X$.

Proof. Let $y \in X$ and let $U$ be an open neighborhood of $y$ in $X$. Then there is a finite collection of indexes $a_{1}, \ldots, a_{n} \in A$ and open sets $V_{a_{1}} \subseteq X_{a_{1}}, \ldots, V_{a_{n}} \subseteq X_{a_{n}}$ such that

$$
y \in p_{a_{1}}^{-1}\left(V_{a_{1}}\right) \cap \cdots \cap p_{a_{n}}^{-1}\left(V_{a_{n}}\right) \subseteq U .
$$

For each $i=1, \ldots, n$ choose an $\eta_{i}>0$ such that

$$
\left\{t \in X_{a_{l}} \mid d\left(t, y_{a_{l}}\right) \leq \eta_{i}\right\} \subseteq V_{a_{l}}, \quad i=1, \ldots, n .
$$

Let $a^{\prime} \geq a_{1}, \ldots, a_{n}$ satisfy (A2) and (A3) for each $a_{i}, i=1, \ldots, n$, and $\eta_{i} / 5$ (see Remark 1). Moreover, let $a^{\prime}$ satisfy (L) for $y, a_{i}$ and $\eta_{i} / 5, i=1, \ldots, n$, so that

$$
d\left(y_{a_{i}}, p_{a_{1} a^{\prime}}\left(y_{a^{\prime}}\right)\right) \leq \eta_{i} / 5, \quad i=1, \ldots, n .
$$

Let $V_{a^{\prime}}=N\left(y_{a^{\prime}}, \varepsilon_{a^{\prime}}\right)$. We claim that

$$
y \in p_{a^{\prime}}^{-1}\left(V_{a^{\prime}}\right) \subseteq p_{a_{1}}^{-1}\left(V_{a_{1}}\right) \cap \cdots \cap p_{a_{n}}^{-1}\left(V_{a_{n}}\right) .
$$

It suffices to show that $x \in p_{a^{\prime}}^{-1}\left(V_{a^{\prime}}\right)$, i.e.,

$$
d\left(x_{a^{\prime}}, y_{a^{\prime}}\right)<\varepsilon_{a^{\prime}}
$$

implies

$$
d\left(x_{a_{l}}, y_{a_{t}}\right) \leq \eta_{i}, \quad i=1, \ldots, n .
$$

Notice that (L) applied to $x$ yields an $a^{\prime \prime} \geq a^{\prime}$ such that

$$
\begin{gathered}
d\left(p_{a_{1} a^{\prime \prime}}\left(x_{a^{\prime \prime}}\right), x_{a_{l}}\right) \leq \eta_{i} / 5, \quad i=1, \ldots, n, \\
d\left(p_{a^{\prime} a^{\prime \prime}}\left(x_{a^{\prime \prime}}\right), x_{a^{\prime}}\right) \leq \varepsilon_{a^{\prime}} .
\end{gathered}
$$


By the choice of $a^{\prime}$ we have

$$
d\left(p_{a_{t} a^{\prime}} p_{a^{\prime} a^{\prime \prime}}\left(x_{a^{\prime \prime}}\right), p_{a_{i} a^{\prime \prime}}\left(x_{a^{\prime \prime}}\right)\right) \leq \eta_{i} / 5, \quad i=1, \ldots, n .
$$

Moreover, (8) and (5) imply (by (A3))

$$
\begin{gathered}
d\left(p_{a_{i} a^{\prime}} p_{a^{\prime} a^{\prime \prime}}\left(x_{a^{\prime \prime}}\right), p_{a_{i} a^{\prime}}\left(x_{a^{\prime}}\right)\right) \leq \eta_{i} / 5, \quad i=1, \ldots, n . \\
d\left(p_{a_{1} a^{\prime}}\left(x_{a^{\prime}}\right), p_{a_{i} a^{\prime}}\left(y_{a^{\prime}}\right)\right) \leq \eta_{i} / 5, \quad i=1, \ldots, n .
\end{gathered}
$$

Now (7), (9), (10), (11) and (3) imply (6) as desired.

LeMma 4. For every $a \in A$ and $\eta>0$ there is an $a^{\prime} \geq a$ such that for every $a^{\prime \prime} \geq a^{\prime}$ one has

$$
d\left(p_{a a^{\prime \prime}} p_{a^{\prime \prime}}, p_{a}\right) \leq \eta
$$

Proof.. Choose $a^{\prime} \geq a$ so that (A2) and (A3) hold for $a$ and $\eta / 3$. Let $a^{\prime \prime} \geq a^{\prime}$ and let $x \in X$. By (L), for any sufficiently large $a^{*} \geq a^{\prime \prime}$ one has

$$
\begin{gathered}
d\left(x_{a}, p_{a a^{*}}\left(x_{a^{*}}\right)\right) \leq \eta / 3, \\
d\left(x_{a^{\prime \prime}}, p_{a^{\prime \prime} a^{*}}\left(x_{a^{*}}\right)\right) \leq \varepsilon_{a^{\prime \prime}} .
\end{gathered}
$$

By (14) and the choice of $a^{\prime}$,

$$
\begin{aligned}
& d\left(p_{a a^{\prime \prime}}\left(x_{a^{\prime \prime}}\right), p_{a a^{\prime \prime}} p_{a^{\prime \prime} a^{*}}\left(x_{a^{*}}\right)\right) \leq \eta / 3, \\
& d\left(p_{a a^{\prime \prime}} p_{a^{\prime \prime} a^{*}}\left(x_{a^{*}}\right), p_{a a^{*}}\left(x_{a^{*}}\right)\right) \leq \eta / 3 .
\end{aligned}
$$

Now, (13), (16) and (15) yield the desired inequality

$$
d\left(p_{a a^{\prime \prime}} p_{a^{\prime \prime}}(x), p_{a}(x)\right)=d\left(p_{a a^{\prime \prime}}\left(x_{a^{\prime \prime}}\right), x_{a}\right) \leq \eta \text {. }
$$

Proof of Theorem 3. There is no loss of generality in assuming that $\mathscr{U}$ consists of $n$ sets of the form $p_{a_{l}}^{-1}\left(V_{i}\right), i=1, \ldots, n$, where $a_{i} \in A$ and $V_{i} \subseteq X_{a_{i}}$ is open (Lemma 3). Choose closed sets $F_{i} \subseteq X, i=1, \ldots, n$, such that

$$
F_{i} \subseteq p_{a_{l}}^{-1}\left(V_{i}\right), \quad i=1, \ldots, n,
$$

and that $\left\{F_{1}, \ldots, F_{n}\right\}$ covers $X$. Next choose closed sets $H_{i} \subseteq X_{a_{i}}$ such that $H_{i} \subseteq V_{i}$ and

$$
F_{i} \subseteq p_{a_{i}}^{-1}\left(H_{i}\right) \subseteq p_{a_{i}}^{-1}\left(V_{i}\right)
$$


Finally, choose numbers $\eta_{i}>0, i=1, \ldots, n$, such that the $\eta_{i}$-neighborhood of $H_{i}$

$$
N\left(H_{i}, \eta_{i}\right) \subseteq V_{i}
$$

By Lemma 4, there is an $a \geq a_{1}, \ldots, a_{n}$ such that

$$
d\left(p_{a_{1}}, p_{a_{1} a} p_{a}\right)<\eta_{i} / 2, \quad i=1, \ldots, n .
$$

Now consider the sets

$$
\begin{gathered}
W_{i}=N\left(H_{i}, \eta_{i} / 2\right), \\
G_{i}=p_{a_{1} a}^{-1}\left(W_{i}\right), \quad i=1, \ldots, n .
\end{gathered}
$$

We claim that

$$
p_{a_{i}}^{-1}\left(H_{i}\right) \subseteq p_{a}^{-1}\left(G_{i}\right) \subseteq p_{a_{i}}^{-1}\left(V_{i}\right), \quad i=1, \ldots, n .
$$

Indeed if $x \in p_{a_{l}}^{-1}\left(H_{i}\right)$, then (21) and (22) imply

$$
p_{a_{1} a} p_{a}(x) \in W_{i}, \quad i=1, \ldots, n .
$$

Now (25) and (23) imply $p_{a}(x) \in G_{i}$, i.e., $x \in p_{a}^{-1}\left(G_{i}\right)$, which establishes the first inclusion in (24).

In order to establish the second inclusion in (24) consider $x \in$ $p_{a}^{-1}\left(G_{i}\right)$. Clearly $p_{a}(x) \in G_{i}=p_{a_{i} a}^{-1}\left(W_{i}\right)$, and thus

$$
p_{a_{i} a} p_{a}(x) \in N\left(H_{i}, \eta_{i} / 2\right) \text {. }
$$

Using (21), we conclude that

$$
p_{a_{t}}(x) \in N\left(H_{i}, \eta_{i}\right) \subseteq V_{i},
$$

i.e., $x \in p_{a_{i}}^{-1}\left(V_{i}\right)$ as desired.

Since $\left\{F_{1}, \ldots, F_{n}\right\}$ is a covering of $X,(19)$ and (24) show that $\left\{p_{a}^{-1}\left(G_{1}\right), \ldots, p_{a}^{-1}\left(G_{n}\right)\right\}$ is an open covering of $X$ which refines $\mathscr{U}$. Therefore, $\mathscr{V}=\left\{G_{1}, \ldots, G_{n}, X_{a} \backslash p_{a}(X)\right\}$ is an open covering of $X_{a}$ which has the desired property that $p_{a}^{-1}(\mathscr{V})$ refines $\mathscr{U}$.

The next theorem is an easy consequence of Theorem 3 .

THEOREM 4. Let $\mathbf{X}=\left(X_{a}, \varepsilon_{a}, p_{a a^{\prime}}, A\right)$ be an approximate inverse system of metric compacta with limit $X$. If $\operatorname{dim} X_{a} \leq n$ for each $a \in A$, then also $\operatorname{dim} X \leq n$.

Proof. Let $\mathscr{U}$ be an open covering of $X$. By Theorem 3, there is an $a \in A$ and an open covering $\mathscr{V}$ of $X_{a}$ such that $p_{a}^{-1}(\mathscr{V})$ refines $\mathscr{U}$. 
Since $\operatorname{dim} X_{a} \leq n$, there is an open covering $\mathscr{W}$ of $X_{a}$, which refines $\mathscr{V}$ and is of order $\leq n+1$. Clearly $p_{a}^{-1}(\mathscr{W})$ refines $\mathscr{U}$ and is of order $\leq n+1$, which proves that $\operatorname{dim} X \leq n$.

REMARK 3. Having in mind applications of approximate inverse systems of compact polyhedra, we have stated our definitions and proved our theorems for approximate inverse systems of metric compacta. However they generalize in a straightforward way to the case of approximate systems of compact Hausdorff spaces. The numbers $\varepsilon_{a}$ and $\eta$ must be replaced by open coverings $\mathscr{U}_{a}, \mathscr{V}$. Conditions of the form $d(f, g) \leq \varepsilon$ become $(f, g) \leq \mathscr{V}$ and mean that the maps $f, g$ are $\mathscr{V}$-near. All our theorems remain true. The obvious changes in the proofs require use of iterated star-refinements of the given covers.

\section{The expansion theorem.}

THEOREM 5. let $X$ be a compact Hausdorff space of covering dimension $\operatorname{dim} X \leq n$. Then there exists an approximate inverse system of compact polyhedra $\mathbf{P}=\left(P_{a}, \varepsilon_{a}, p_{a a^{\prime}}, A\right)$ such that $\operatorname{dim} P_{a} \leq n$ and the limit $P=\lim \mathbf{P}$ is homeomorphic to $X$. Moreover, $\operatorname{card}(A) \leq$ weight $(X)$.

In view of the negative results stated in $\S 1$ this expansion theorem demonstrates the significance of this new concept of approximate systems. It also shows that in general there is no way to modify an approximate system (keeping its members) so as to transform it into a commutative system with a homeomorphic limit.

The proof of Theorem 5 is divided into 5 parts.

5.1. Construction of $\mathbf{P}$. By the Tihonov embedding theorem there is an embedding $e: X \rightarrow Y$ of $X$ into an infinite cube $Y=I^{\tau}$ where $\tau=\operatorname{weight}(X)$. On the other hand the cube $Y$ is the inverse limit of an inverse system $\mathbf{Y}=\left(Y_{a}, q_{a a^{\prime}}, A\right)$ of finite-dimensional cubes $Y_{a}$, where $A$ is the set of all finite subsets of a set of cardinality $\tau$. Note that $A$ is cofinite (order by inclusion) and $\operatorname{card}(A) \leq$ weight $(X)$. Let $q_{a}: Y \rightarrow Y_{a}$ be the natural projections and let $|a| \geq 0$ denote the number of predecessors of $a \in A$. We will define, by induction on $|a|$, the following data: for each $a \in A$, a compact polyhedron $P_{a}$, $\operatorname{dim} P_{a} \leq n$, maps $g_{a}: X \rightarrow P_{a}, h_{a}: P_{a} \rightarrow Y_{a}$ and numbers $\varepsilon_{a}>0$, $\delta_{a}>0$ and for each pair $a \leq a^{\prime}$ a map $p_{a a^{\prime}}: P_{a^{\prime}} \rightarrow P_{a}$. We require that 
each $g_{a}: X \rightarrow P_{a}$ be surjective and that the following conditions hold.

(4) $x, x^{\prime} \in P_{a^{\prime}}, \quad d\left(x, x^{\prime}\right) \leq \varepsilon_{a^{\prime}} \Rightarrow d\left(p_{a a^{\prime}}(x), p_{a a^{\prime}}\left(x^{\prime}\right)\right) \leq \varepsilon_{a} / 3^{\left|a^{\prime}\right|-|a|}$,

(5) $y, y^{\prime} \in Y_{a^{\prime}}, \quad d\left(y, y^{\prime}\right) \leq \delta_{a^{\prime}} \Rightarrow d\left(q_{a a^{\prime}}(y), q_{a a^{\prime}}\left(y^{\prime}\right)\right) \leq \delta_{a} / 3^{\left|a^{\prime}\right|-|a|}$.

The construction of such data is possible due to the following lemma, proved in [4] (as Lemma 2).

LeMmA 5. Let $X$ be a compact Hausdorff space with $\operatorname{dim} X \leq n$. Let $P_{1}, \ldots, P_{k}$ be compact polyhedra, let $\varepsilon_{1}>0, \ldots, \varepsilon_{k}>0$ and let $f_{1}: X \rightarrow P_{1}, \ldots, f_{k}: X \rightarrow P_{k}$ be maps. Then there exist a compact polyhedron $P, \operatorname{dim} P \leq n$, a surjective map $g: X \rightarrow P$ and maps $p_{1}: P \rightarrow$ $P_{1}, \ldots, p_{k}: P \rightarrow P_{k}$, such that $d\left(f_{1}, p_{1} g\right) \leq \varepsilon_{1}, \ldots, d\left(f_{k}, p_{k} g\right) \leq \varepsilon_{k}$.

We now assume that we have already defined $P_{a}, g_{a}, h_{a}, \varepsilon_{a}, \delta_{a}$ and $p_{a_{1} a}$ for $|a| \leq m$ and we assume that $\left|a^{\prime}\right|=m+1$. Let $a_{1}, \ldots, a_{k}$ be all the predecessors of $a^{\prime}$ (different from $a^{\prime}$ ). We first choose $\delta_{a^{\prime}}$ so that (5) is satisfied (uniform continuity). We then apply Lemma 5 to polyhedra $P_{a_{1}}, \ldots, P_{a_{k}}, Y_{a^{\prime}}$, to numbers

$$
\frac{\varepsilon_{a_{1}}}{3\left|a^{\prime}\right|-\left|a_{1}\right|}, \ldots, \frac{\varepsilon_{a_{k}}}{3\left|a^{\prime}\right|-\left|a_{k}\right|}, \quad \frac{\delta_{a^{\prime}}}{3}
$$

and to maps $g_{a_{1}}, \ldots, g_{a_{k}}, q_{a^{\prime}}$ e. We obtain a compact polyhedron $P=$ $P_{a^{\prime}}, \operatorname{dim} P_{a^{\prime}} \leq n$, a surjection $g_{a^{\prime}}: X \rightarrow P_{a^{\prime}}$, a map $h_{a^{\prime}}: P_{a^{\prime}} \rightarrow Y_{a^{\prime}}$ and maps $p_{a_{1} a^{\prime}}: P_{a^{\prime}} \rightarrow P_{a_{1}}, i=1, \ldots, k$, such that (1) and (2) hold. Finally we choose $\varepsilon_{a^{\prime}}$ so that (3) and (4) are satisfied.

5.2. Verification of (A1)-(A3). We will now show that $\mathbf{P}=\left(P_{a}, \varepsilon_{a}\right.$, $\left.p_{a a^{\prime}}, A\right)$ is an approximate system. Indeed, if $a<a^{\prime}<a^{\prime \prime}$, then for every $x \in X$ we have by (1)

$$
d\left(g_{a^{\prime}}(x), p_{a^{\prime} a^{\prime \prime}} g_{a^{\prime \prime}}(x)\right) \leq \varepsilon_{a^{\prime}},
$$

which by (4) implies

$$
d\left(p_{a a^{\prime}} g_{a^{\prime}}(x), p_{a a^{\prime}} p_{a^{\prime} a^{\prime \prime}} g_{a^{\prime \prime}}(x)\right) \leq \varepsilon_{a} / 3^{\left|a^{\prime}\right|-|a|} \leq \varepsilon_{a} / 3 .
$$

Also by (1) we have

$$
\begin{aligned}
& d\left(g_{a}(x), p_{a a^{\prime}} g_{a^{\prime}}(x)\right) \leq \varepsilon_{a} / 3^{\left|a^{\prime}\right|-|a|} \leq \varepsilon_{a} / 3, \\
& d\left(g_{a}(x), p_{a a^{\prime \prime}} g_{a^{\prime \prime}}(x)\right) \leq \varepsilon_{a} / 3^{\left|a^{\prime \prime}\right|-|a|} \leq \varepsilon_{a} / 3 .
\end{aligned}
$$


Now (7), (8), (9) yield

$$
d\left(p_{a a^{\prime}} p_{a^{\prime} a^{\prime \prime}} g_{a^{\prime \prime}}(x), p_{a a^{\prime \prime}} g_{a^{\prime \prime}}(x)\right) \leq \varepsilon_{a} .
$$

Since $g_{a^{\prime \prime}}: X \rightarrow P_{a^{\prime \prime}}$ is onto, (10) proves (A1).

In order to prove (A2) choose $n$ so large that $3 \varepsilon_{a} / 3^{n} \leq \eta$ and choose $a^{\prime}>a$ such that $\left|a^{\prime}\right| \geq|a|+n$. Let $a_{2}>a_{1} \geq a^{\prime}$. Replacing in (7), (8), (9) $a^{\prime}, a^{\prime \prime}$ by $a_{1}, a_{2}$, we see that

$$
\begin{aligned}
d\left(p_{a a_{1}} p_{a_{1} a_{2}} g_{a_{2}}(x), p_{a a_{2}} g_{a_{2}}(x)\right) & \leq 2 \varepsilon_{a} / 3^{\left|a_{1}\right|-|a|}+\varepsilon_{a} / 3^{\left|a_{2}\right|-|a|} \\
& \leq 3 \varepsilon_{a} / 3^{n} \leq \eta,
\end{aligned}
$$

which establishes (A2).

Finally, for the same choice of $a^{\prime}$ and $a^{\prime \prime} \geq a^{\prime}$, we see that by (4), $x, x^{\prime} \in P_{a^{\prime \prime}}, d\left(x, x^{\prime}\right) \leq \varepsilon_{a^{\prime \prime}}$ implies

$$
d\left(p_{a a^{\prime \prime}}(x), p_{a a^{\prime \prime}}\left(x^{\prime}\right)\right) \leq \varepsilon_{a} / 3^{\left|a^{\prime \prime}\right|-|a|} \leq \eta / 3,
$$

which establishes (A3).

5.3. The map $g: X \rightarrow P$. Let $P$ be the limit of $\mathbf{P}$ and let $p_{a}: P \rightarrow P_{a}$ be the corresponding projections. We will now define a homeomorphism $g: X \rightarrow P$.

If $x \in X$, by (1), the points $g_{a}(x) \in P_{a}, a \in A$, form a prethread for P. Let $z=\left(z_{a}\right)$ be the thread generated by this prethread (see Lemma $2)$. Then $z_{a}=\lim _{a^{\prime}} p_{a a^{\prime}} g_{a^{\prime}}(x)$. We now define $g$ by putting $g(x)=z$, i.e.,

$$
(g(x))_{a}=\lim _{a^{\prime}} p_{a a^{\prime}} g_{a^{\prime}}(x) .
$$

In order to show that $g$ is continuous, it suffices to show that the map $x \mapsto(g(x))_{a}=z_{a}$ is continuous for each $a \in A$.

Given a point $x \in X$ and an $\eta>0$, we choose by the continuity of $g_{a}$, a neighborhood $U$ of $X$ in $X$ so small that

$$
x^{\prime} \in U \Rightarrow d\left(g_{a}\left(x^{\prime}\right), g_{a}(x)\right) \leq \eta / 3 .
$$

We assert that

$$
x^{\prime} \in U \Rightarrow d\left(g\left(x^{\prime}\right)_{a}, g(x)_{a}\right) \leq \eta .
$$

Indeed, choose $a^{\prime} \geq a$ so large that $\varepsilon_{a} / 3^{\left|a^{\prime}\right|-|a|} \leq \eta / 3$. Then by (1), $a^{\prime \prime} \geq a^{\prime}$ implies

$$
d\left(p_{a a^{\prime \prime}} g_{a^{\prime \prime}}, g_{a}\right) \leq \varepsilon_{a} / 3^{\left|a^{\prime \prime}\right|-|a|} \leq \eta / 3 .
$$


Therefore, for $x^{\prime} \in U$, we have

$$
\begin{aligned}
& d\left(p_{a a^{\prime \prime}} g_{a^{\prime \prime}}(x), p_{a a^{\prime \prime}} g_{a^{\prime \prime}}\left(x^{\prime}\right)\right) \\
& \quad \leq d\left(p_{a a^{\prime \prime}} g_{a^{\prime \prime}}(x), g_{a}(x)\right)+d\left(g_{a}(x), g_{a}\left(x^{\prime}\right)\right) \\
& \quad+d\left(g_{a}\left(x^{\prime}\right), p_{a a^{\prime \prime}} g_{a^{\prime \prime}}\left(x^{\prime}\right)\right) \leq \eta .
\end{aligned}
$$

Passing to the limit with $a^{\prime \prime}$ in (17) we obtain (15), as desired.

5.4. $g$ is injective. Consider any two distinct points $x, x^{\prime} \in X$. We must show that $g(x) \neq g\left(x^{\prime}\right)$.

Since $e: X \rightarrow Y$ is an embedding, we have $e(x) \neq e\left(x^{\prime}\right)$. Therefore, there is an index $a \in A$ such that

$$
q_{a} e(x) \neq q_{a} e\left(x^{\prime}\right) .
$$

Choose a number $\eta>0$ such that

$$
d\left(q_{a} e(x), q_{a} e\left(x^{\prime}\right)\right)>\eta>0 .
$$

We claim that for each sufficiently large $a^{\prime} \geq a$ one has

$$
d\left(q_{a a^{\prime}} h_{a^{\prime}} g_{a^{\prime}}(x), q_{a a^{\prime}} h_{a^{\prime}} g_{a^{\prime}}\left(x^{\prime}\right)\right)>\eta / 3 .
$$

Indeed, for $a^{\prime} \geq a$ we have

$$
\begin{aligned}
d\left(q_{a} e(x), q_{a} e\left(x^{\prime}\right)\right) \leq & d\left(q_{a} e(x), q_{a a^{\prime}} h_{a^{\prime}} g_{a^{\prime}}(x)\right) \\
& +d\left(q_{a a^{\prime}} h_{a^{\prime}} g_{a^{\prime}}(x), q_{a a^{\prime}} h_{a^{\prime}} g_{a^{\prime}}\left(x^{\prime}\right)\right) \\
& +d\left(q_{a a^{\prime}} h_{a^{\prime}} g_{a^{\prime}}\left(x^{\prime}\right), q_{a} e\left(x^{\prime}\right)\right) .
\end{aligned}
$$

Moreover, by (2) for $a^{\prime}$ and (5), we have

$$
d\left(q_{a a^{\prime}} q_{a^{\prime}} e, q_{a a^{\prime}} h_{a^{\prime}} g_{a^{\prime}}\right) \leq \delta_{a} / 3^{\left|a^{\prime}\right|-|a|} .
$$

Therefore, for all sufficiently large $a^{\prime}$ the first and the third term on the right side of $(21)$ are $\leq \eta / 3$. If the same were true for the middle term, (21) would contradict (19).

Now note that (5) and (20) imply

$$
d\left(h_{a^{\prime}} g_{a^{\prime}}(x), h_{a^{\prime}} g_{a^{\prime}}\left(x^{\prime}\right)\right)>\delta_{a^{\prime}}
$$

for sufficiently large $a^{\prime}$. Furthermore, (3) for $a^{\prime}$ and (23) imply

$$
d\left(g_{a^{\prime}}(x), g_{a^{\prime}}\left(x^{\prime}\right)\right)>\varepsilon_{a^{\prime}} .
$$

Our next claim is that

$$
d\left(p_{a^{\prime} a^{\prime \prime}} g_{a^{\prime \prime}}(x), p_{a^{\prime} a^{\prime \prime}} g_{a^{\prime \prime}}\left(x^{\prime}\right)\right)>\varepsilon_{a^{\prime}} / 3
$$


for all sufficiently large $a^{\prime}$ and all $a^{\prime \prime}>a^{\prime}$. Indeed,

(26) $d\left(g_{a^{\prime}}(x), g_{a^{\prime}}\left(x^{\prime}\right)\right)$

$$
\begin{aligned}
& \leq d\left(g_{a^{\prime}}(x), p_{a^{\prime} a^{\prime \prime}} g_{a^{\prime \prime}}(x)\right)+d\left(p_{a^{\prime} a^{\prime \prime}} g_{a^{\prime \prime}}(x), p_{a^{\prime} a^{\prime \prime}} g_{a^{\prime \prime}}\left(x^{\prime}\right)\right) \\
& \quad+d\left(p_{a^{\prime} a^{\prime \prime}} g_{a^{\prime \prime}}\left(x^{\prime}\right), g_{a^{\prime}}\left(x^{\prime}\right)\right) .
\end{aligned}
$$

By (1), the first and the last terms on the right side of $(26)$ are $\leq \varepsilon_{a^{\prime}} / 3$. If the middle term were also $\leq \varepsilon_{a^{\prime}} / 3,(26)$ would contradict (24).

Passing to the limit with $a^{\prime \prime}$ in (25), one obtains

$$
d\left((g(x))_{a^{\prime}},\left(g\left(x^{\prime}\right)\right)_{a^{\prime}}\right) \geq \varepsilon_{a^{\prime}} / 3>0,
$$

for all sufficiently large $a^{\prime}$. Consequently $(g(x))_{a^{\prime}} \neq\left(g\left(x^{\prime}\right)\right)_{a^{\prime}}$ and thus $g(x) \neq g\left(x^{\prime}\right)$, as desired.

5.5. $g$ is surjective. Since $X$ is compact, it suffices to show that every $z \in P$ is in the closure of $g(X)$, i.e., that $g(X)$ meets every neighborhood $U$ of $z$. By Lemma 3, we can assume that $U$ is of the form $p_{a}^{-1}(V)$ where $a \in A$ and $V \subseteq P_{a}$ is an open neighborhood of $z_{a}$. Clearly there is an $\eta>0$ so small that $u \in P$ and $d\left(u_{a}, z_{a}\right) \leq \eta$ imply $u \in p_{a}^{-1}(V)$. Therefore it suffices to produce an $x \in X$ such that

$$
d\left((g(x))_{a}, z_{a}\right) \leq \eta .
$$

Choose $a_{1}>a$ so large that $\varepsilon_{a} / 3^{\left|a_{1}\right|-|a|} \leq \eta / 3$. Using (L) for $z$, choose $a^{\prime} \geq a_{1}$, so large that

$$
d\left(z_{a}, p_{a a^{\prime}}\left(z_{a^{\prime}}\right)\right)<\eta / 3 .
$$

Since $g_{a^{\prime}}: X \rightarrow P_{a^{\prime}}$ is onto, there is an $x \in X$ such that

$$
g_{a^{\prime}}(x)=z_{a^{\prime}} .
$$

For $a^{\prime \prime} \geq a_{1}$, by (1) we have

$$
d\left(p_{a a^{\prime \prime}} g_{a^{\prime \prime}}(x), g_{a}(x)\right) \leq \eta / 3 .
$$

Passing to the limit with $a^{\prime \prime}$ in (31), one obtains

$$
d\left((g(x))_{a}, g_{a}(x)\right) \leq \eta / 3 .
$$

Also, by (1), one has

$$
d\left(g_{a}(x), p_{a a^{\prime}} g_{a^{\prime}}(x)\right) \leq \eta / 3 .
$$

Now (32), (33), (30) and (29) yield the desired formula (28).

This completes the proof of Theorem 5 . 


\section{REFERENCES}

[1] S. Eilenberg and N. E. Steenrod, Foundations of Algebraic Topology, Princeton Univ. Press, Princeton 1952.

[2] H. Freudenthal, Entwicklungen von Räumen und ihren Gruppen, Compositio Math., 4 (1937), 145-234.

[3] S. Mardešić, Chainable continua and inverse limits, Glasnik Mat-Fiz. Astron., 14 (1959), 219-232.

[4] On covering dimension and inverse limits of compact spaces, Illinois J. Math., 4 (1960), 278-291.

[5] S. Mardešić and T. Watanabe, Strong homology and dimension, Topology Appl., 29 (1988), 185-205.

[6] B. A. Pasynkov, On polyhedral spectra and dimension of bicompacta and of bicompact groups (Russian), Dokl. Akad. Nauk USSR, 121, No. 1 (1958), 4548.

[7] On spectra and dimension of topological spaces (Russian), Mat. Sbornik, 57 (99), No. 4 (1962), 449-476.

Received May 13, 1987 and in revised form May 3, 1988. This paper was written during February-March of 1987 while S. Mardešić, on leave from the University of Zagreb, was visiting the University of Oklahoma in Norman, Oklahoma.

UNIVERSITY OF ZAGREB

41001 ZAGREB, P.O. BOX 187

YugosLavia

AND

UNIVERSITY OF OKLAHOMA

NORMAN, OK 73019

U.S.A. 